\title{
Kinematic Analysis of the Tram-pedestrian Collision - a Preliminary Case Study
}

Lubos Tomsovsky (000-0003-0047-6028)1, Petr Kubovy (0000-0001-7634-2910), Frantisek Lopot (0000-00019955-0261) ${ }^{1}$, Karel Jelen (0000-0002-8151-9810) ${ }^{1}$

Faculty of Physical Education and Sport, Charles University in Prague. José Martího 31, 16252 Prague 6. Czech Republic. E-mails: lubostomsovsky@gmail.com, kubovy@ftvs.cuni.cz,lopot@ftvs.cuni.cz, jelen@ftvs.cuni.cz.

The goal of the study was to conduct a preliminary analysis of the kinematics of tram-pedestrian collision in case of a side impact. A T6A5-type tram, travelling at the speed of $10 \mathrm{~km} / \mathrm{h}$, was used for the analysis and reconstruction of the collision. The pedestrian response was analysed using a crash-test dummy. The postimpact dummy movement was approximated by the motion of its centre of mass (COM) and only the translation movement in the frontal plane of dummy (along the horizontal and vertical axes) was considered. The results showed a significant change of coefficient of restitution after the initial impact. At first, the coefficient of restitution was high $(e=0.94)$, suggesting an almost perfectly elastic collision, followed by a sharp decrease $(e=0.07)$ within a short period of time $(t=0.02 s)$ that suggested an almost perfectly inelastic collision. After that, the coefficient of restitution reached a plateau phase with the values ranging from 0.18 to 0.32 and corresponding to a percentage loss of kinetic energy falling within $89 \%$ and $97 \%$. The preliminary analysis of this study highlighted some features of sideimpact tram-pedestrian collision and its kinematics.

Keywords: Tram, Pedestrian, Collision, Coefficient of restitution, Safety.

\section{Introduction}

Pedestrians represent the most vulnerable group of participants in traffic accidents and the public transport is not an exception $[1,2]$. Due to a growing volume of public transport, general rush and pressure of modern life, and also pedestrians ' lack of attention around the public transport, the issue of safety has become

important $[1,2,3]$. Although there have been a lot of effort, though, manpower, material, and financial resources put into the understanding of the vehicle-pedestrian collisions, developing and improving novel traffic safety technologies and vehicles' body design by automakers and related research institutions, the number of fatalities and severe injuries has remained high $[1,2,4]$. Therefore, the pedestrian safety and injury risk reduction focused on the public transport in urban areas have become a growing concern and European institutions together with rolling stock manufactures have already started developing the methodology for testing the passive safety of vehicle bodies $[3,5]$.

The kinematic analysis of the motion of colliding bodies before, during, and after the collision is a first step towards the reconstruction of traffic accidents and understanding the mechanical and biomechanical response to the impact [6]. The goal of this study was to provide a preliminary kinematic analysis of tramdummy collision in case of a side impact. A pedestrian dummy was used for the simulation of human body reaction to the impact of a T6A5-type tram (ČKD Tatra, Prague, Czech Republic), typical for Prague public transport, approaching at the speed of $10 \mathrm{~km} / \mathrm{h}$. The analysis was focused on the interaction of the dummy's centre of mass (COM) and front end of the tram from the initial impact to the moment when the tram started to brake. For the purpose of this study, the analysis only included the translational movements in the dummy's frontal plane and rotational movements were neglected. The coefficient of restitution calculations, which are related to the percentage loss in internal kinetic energy of tram-dummy system, were used to provide a different approach towards studying traffic collisions, understanding and improving the reconstruction of such traffic accidents, as well as assisting in the future design and development of novel technologies to improve the pedestrian safety. The results could also improve the study of complex numerical simulations of tram-pedestrian collisions to increase the accuracy of such methods, techniques, and models [7].

\section{Methods}

This case study represents one of the first stages of a long-term research project focused on the tram-pedestrian collisions; the kinematics, dynamics, and biomechanics of such road accidents, their reconstruction, computational modeling and its validaton, and 
injury assessment (type, mechanism, severity). The goal of this part of the project was to provide a preliminary kinematic analysis of the tram-pedestrian collision in case of a side impact. The overall goal of the project is then to provide a better understanding of tram-pedestrian collisions and possibly increase the safety of pedestrians by improving the design of trams or developing new technologies (such as collision avoidance systems). The project is funded by Operational Programme Research, Development and Education CZ.02.1.01/0.0/0.0/16_026/0008401.

\subsection{Instruments}

A T6A5-type tram was used for the purpose of this study. The tram is unidirectional, high-floor, and very common for Prague public transport (Fig. 1). The tram was travelling at the speed of $10 \mathrm{~km} / \mathrm{h}$ at the time of collision. The collision took place in the middle of a $200 \mathrm{~m}$ straight tramway track at the testing facilities of the VUKV a.s. (The Research, Development and Testing of Railway Rolling Stock centre).

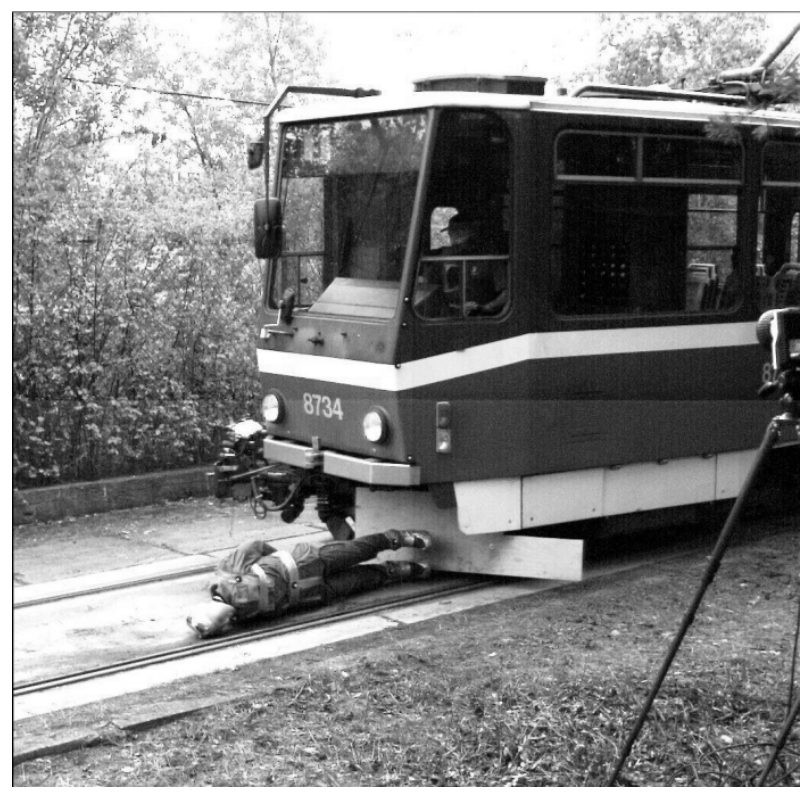

Fig. 1 A T6 A5-type tram (a front end)

The Hybrid III 50th Percentile Male Pedestrian Dummy (JASTI, Tokyo, Japan) was used to replicate the human biomechanical behaviour during the collision. The dummy was placed sideways towards the approaching tram, exactly $0.15 \mathrm{~m} \times$ the width of tram from the centre line towards one end of the track (Fig. 2). This position follows a current version of the European regulation regarding the passive safety of rail

vehicles [5]. The dummy is, however, validated for frontal collision tests only, which is the limitation of the current study. Though at the same time, the verification of the dummy for side-impact collision tests represents another goal of the whole project.

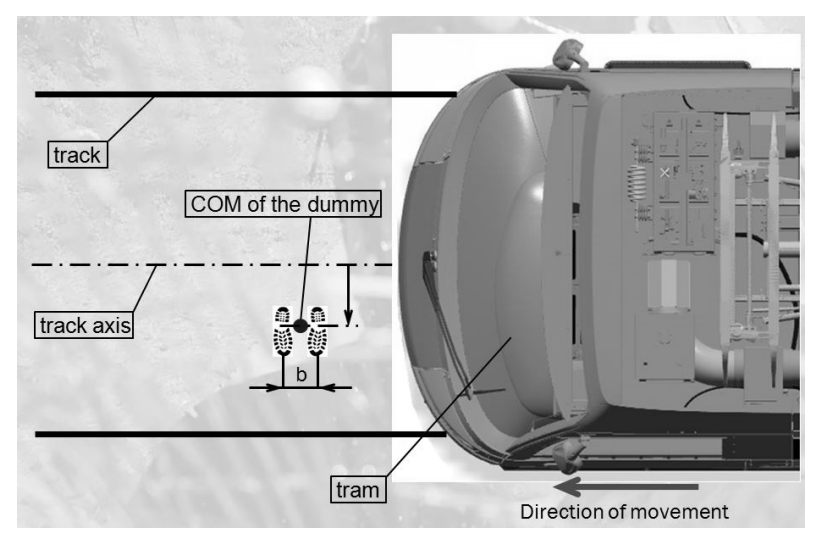

Fig. 2The crash test configuration (side impact, dummy placement in front of the approaching tram)

The kinematics of tram-pedestrian collision was measured with two different technologies. Firstly, two ultra high-speed cameras (Photron, Tokyo, Japan) were used to record the collision itself (frontal view of the dummy and front end of the tram, recording frequency of $12000 \mathrm{~Hz}$ ) and the whole tram-pedestrian system (frontal view of the approaching tram and dummy placed sideways, recording frequency of 500 $H \approx$ ). And secondly, the Qualisys motion capture system (Qualisys AB, Göteborg, Sweden) was utilized to capture the movement of reflective passive markers attached to the dummy and front end of the tram during the collision with eight cameras at the frequency of $300 \mathrm{~Hz}$. The whole measurement configuration is shown in Figure 3.

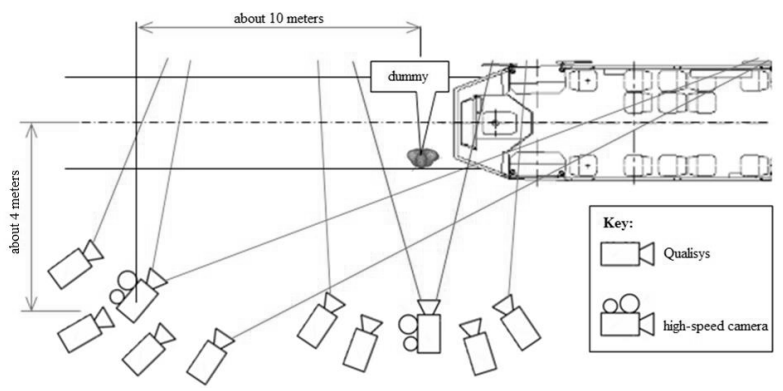

Fig. 3 The measurement configuration (black lines - the tramway tracks, green lines - the area tracked by Qualisys system, red lines - the view of the collision itself recorded by one ultra high-speed camera, blue lines - the whole system of tram and pedestrian recorded by the second ultra high-speed camera)

\subsection{Procedure}

For the purpose of this study, eight passive markers were used to measure the kinematics of the tram-peestrian collision. Six markers were attached to the dummy's head, shoulders, shins, and the area of the centre of mass (COM). The other two markers were attached to the front end of the tram (Fig. 4). The analysis was then focused on the kinematics of the tram and dummy's COM in the frontal plane of the 
dummy and translations along the horizontal and vertical axes were the only movements of interest. Rotational movements were not included in this preliminary analysis as the step-by-step approach was chosen for the whole research project, i.e., from the simplest collision conditions to the most complex ones.

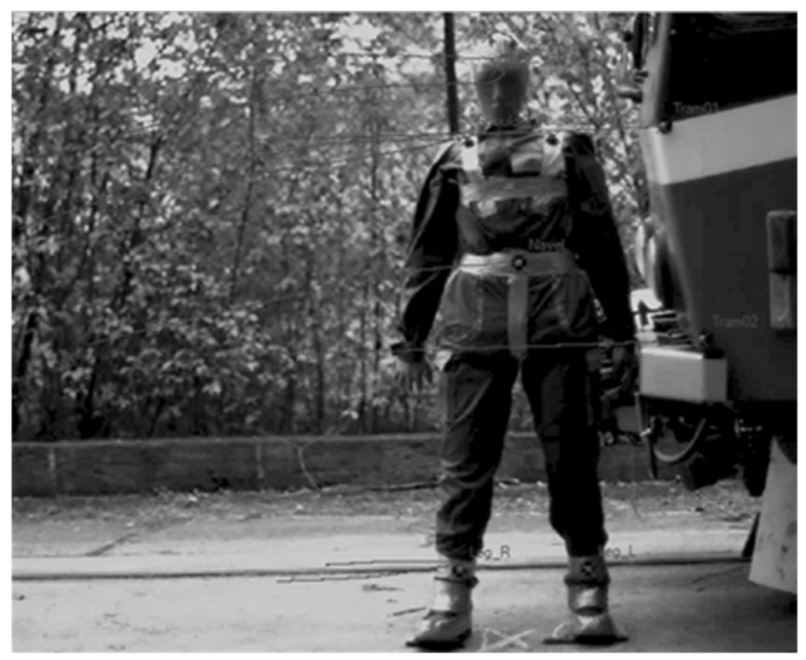

Fig. 4 The frontal view of the collision showing the position of eight passive markers (one on the head, two on shoulders, one in the area of the dummy's COM, two on shins, and two on the front end of tram) and their trajectories

\subsection{Data analysis}

The experimental data were collected using the Qualisys motion capture system at the frequency of $300 \mathrm{~Hz}$. The position and velocity of each marker in the frontal plane of the dummy was analysed and calculated using the Qualisys Track Manager software. The analysis was focused on the position and velocity of the dummy's COM and front end of the tram at every moment before, during, and after the collision until the tram started to brake and the dummy was thrown away in front of the tram. Figure 5 shows the movement of markers during the whole measurement (the position at every moment).

The calculated data were based on several assumptions that allowed describing the events of collision mathematically [8]. The following assumptions were

made for the purpose of this study:

- The side-impact of tram-pedestrian collision is symmetric, i.e., the events of collision take place in one plane (frontal plane of the dummy).

- The initial velocity of dummy is zero.

- The collision is inelastic, i.e., the momentum of tram-pedestrian system is conserved and the internal kinetic energy changes.

- After the launch the dummy is considered as a mass point, only translations are considered, and rotational movements are neglected.

- The collision starts at the moment of initial tram-dummy impact and finishes when the tram starts to brake.

- The ground is flat, friction between the dummy's feet and ground is neglected, as well as all air resistance.

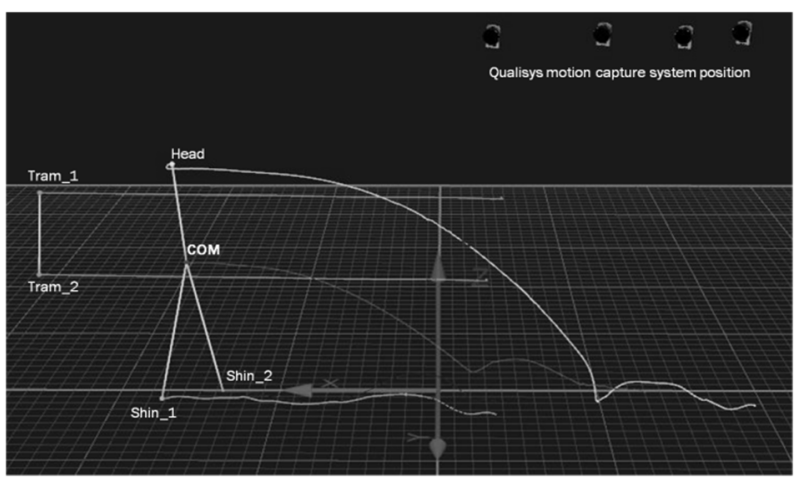

Fig. 5 The movement analysis of each marker during the sideimpact collision using the Qualisys motion capture system

To determine the percentage loss of internal kinetic energy during the impact phase, the equations for the conservation of momentum, coefficient of restitution, and the change of internal kinetic energy were used. The conservation of momentum for inelastic collision in general is:

$$
\begin{aligned}
p_{1}+p_{2} & =p_{1}^{\prime}+p_{2}^{\prime} \\
m_{1} \cdot v_{1}+m_{2} \cdot v_{2} & =m_{1} \cdot v_{1}^{\prime}+m_{2} \cdot v_{2}^{\prime}
\end{aligned}
$$

Where:

$p_{1}, p_{1}^{\prime} \ldots$ Linear momentum of the dummy before and after the collision respectively $\left[\mathrm{kg} \cdot \mathrm{m} \cdot \mathrm{s}^{-1}\right]$,

$p_{2}, p_{2}^{\prime} \ldots$ Linear momentum of the tram before and after the collision respectively $\left[\mathrm{kg} \cdot \mathrm{m} \cdot \mathrm{s}^{-1}\right.$,

$m_{1} \ldots$ Mass of the dummy $[\mathrm{kg}]$,

$m_{2} \ldots$ Mass of the tram $[\mathrm{kg}]$,

$v_{1}, v_{1}$ '...Velocity of the dummy before and after the collision respectively $\left[\mathrm{m} \cdot \mathrm{s}^{-1}\right]$,

$v_{2}, v_{2}^{\prime} \ldots$ Velocity of the tram before and after the collision respectively $\left[m \cdot s^{-1}\right]$.

The mass of dummy was approximately $m_{1}=80$ $\mathrm{kg}$, the mass of tram was $m_{2}=16000 \mathrm{~kg}$, and the initial velocity of dummy (before the collision) was zero $\left(\nu_{1}\right.$ $=0 \mathrm{~m} / \mathrm{s}$ ). The initial velocity of tram was $v_{2}=2.67 \mathrm{~m} / \mathrm{s}$.
The coefficient of restitution $(e)$ was defined as a ratio of the final to the initial relative velocity between the dummy and tram [6]: 


$$
\begin{aligned}
& e=\frac{\text { final relative velocity between the objects }}{\text { initial relative velocity between the objects }} \\
& \qquad e=\left|\frac{v_{2}^{\prime}-v_{1}^{\prime}}{v_{1}-v_{2}}\right|
\end{aligned}
$$

Where:

$e \ldots$ Coefficient of restitution [-]; the value ranges from zero to one.

The coeficient of restitution indicates how much kinetic energy remains after the collision of two objects. If the coefficient of restitution is low (close to zero), the collision is more inelastic and therefore, more kinetic energy is lost in the form of heat, sound, or due to localized deformation. On the other hand, if the coefficient of restitution is high (close to one), the collision is more elastic and therefore, less kinetic energy is lost [8].

Using the equation for conservation of momentum (2) and the equation for coefficient of restitution (4) one can find the velocity of dummy and tram after the collision in general:

$$
\begin{aligned}
& v_{1}^{\prime}=v_{1} \cdot \frac{\left(m_{1}-e \cdot m_{2}\right)}{\left(m_{1}+m_{2}\right)}+v_{2} \cdot \frac{m_{2} \cdot(1+e)}{\left(m_{1}+m_{2}\right)} \\
& v_{2}^{\prime}=v_{1} \cdot \frac{m_{1} \cdot(1+e)}{\left(m_{1}+m_{2}\right)}+v_{2} \cdot \frac{\left(m_{2}-e \cdot m_{1}\right)}{\left(m_{1}+m_{2}\right)}
\end{aligned}
$$

The change of internal kinetic energy $(\Delta K E)$ can be generally described as the difference between the in- ternal kinetic energy of the tram-dummy system before and after the collision:

$$
\begin{gathered}
\Delta K E=K E_{\text {int }}-K E_{\text {int }}^{\prime} \\
\Delta K E=\left(\frac{1}{2} m_{1} \cdot v_{1}^{2}+\frac{1}{2} m_{2} \cdot v_{2}^{2}\right)-\left(\frac{1}{2} m_{1} \cdot\left(v_{1}^{\prime}\right)^{2}+\frac{1}{2} m_{2} \cdot\left(v_{2}^{\prime}\right)^{2}\right)
\end{gathered}
$$

Where:

$\triangle K E$...Change of internal kinetic energy of the tram-dummy system [J],

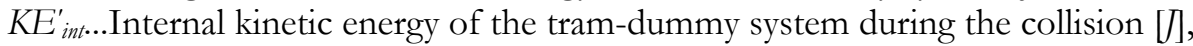

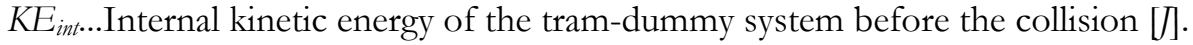

Based on the assumption that the initial velocity of dummy before the collision was zero $\left(v_{1}=0 \mathrm{~m} / \mathrm{s}\right)$, and using the equations for final velocities of dummy and tram $(5,6)$, as well as the equation to determine the coefficient of restitution (4), the formula for change of internal kinetic energy of the tram-dummy system could be derived for the purposes of this study:

$$
\Delta K E=\frac{1}{2} \cdot \frac{m_{1} \cdot m_{2}}{m_{1}+m_{2}} \cdot v_{2}^{2} \cdot\left(1-e^{2}\right)
$$

Finally, the percentage loss of internal kinetic energy during the impact was [6]:

Where:

$$
\text { \%loss in } K E=\frac{\Delta K E}{K E_{\text {int }}} \cdot 100=\frac{\frac{1}{2} \cdot \frac{m_{1} \cdot m_{2}}{\left(m_{1}+m_{2}\right)} \cdot v_{2}^{2} \cdot\left(1-e^{2}\right)}{K E_{\text {int }}} \cdot 100
$$

$\%$ loss in KE...Percentage loss of internal kinetic energy during the impact phase [\%].

As this study was focused only on one specific case of the tram-pedestrian collision using data from just one measurement, a thorough statistical analysis could not be conducted.

\section{Results}

The analysis was focused on kinematics of the tram-dummy system from the first impact until the moment when the tram started to brake. Figure 6 shows a velocity-time graph of the magnitude of velocity, i.e., the speed, of the dummy's COM and front end of the tram during the whole measurement. The graph also includes three phases of the collision (preimpact, post-impact, flying phase) separated by the dashed lines that indicates the collision phase change. The lines also define the area of interest of this study. 


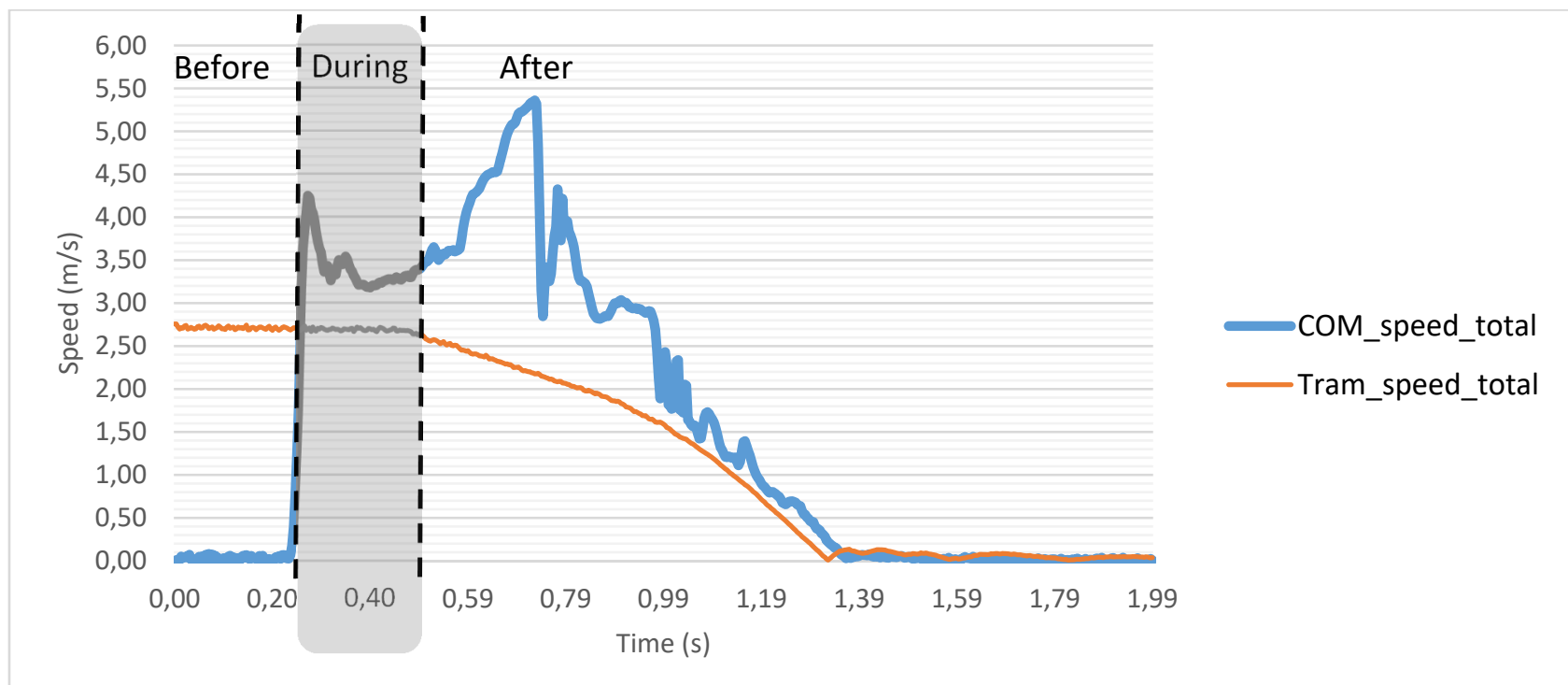

Fig. 6 A velocity-time graph of the speed of dummy's COM and front end of the tram with three phases of the collision (pre-impact, post-impact, flying phase) separated by the dashed lines

After the initial impact, there was a sharp increase of the dummy's COM speed reaching the peak of 4.24 $\mathrm{m} / \mathrm{s}$ followed by a steep drop and a gradual rise right after. Based on the equation for the velocity of dummy after the collision (5), considering almost perfectly elastic collision after the initial impact $(e \approx 1)$, and considering the mass of dummy was small enough to be negligible compared to the mass of tram, the magnitude of dummy's velocity can reach a double of tram's speed before the collision $\left(v_{2}=2.67 \mathrm{~m} / \mathrm{s} \rightarrow\right.$ $\left.v_{1}{ }_{\max }=5.34 \mathrm{~m} / \mathrm{s}\right)$. The whole phenomenon after the initial impact could be caused by the kinetic energy transfer from the tram to dummy and the contribution of movements of other parts of the dummy and their inertia. The results also showed that the reaction time of a driver to start to brake was almost exactly $0.25 \mathrm{~s}$. After the braking point, the dummy's COM was accelerated mainly due to the contribution summation of movements of other body parts.

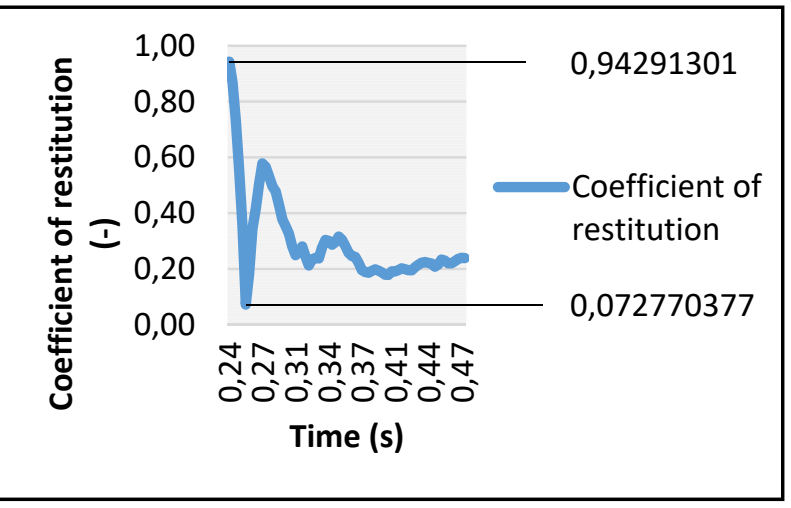

Fig. 7 The change of coefficient of restitution for every time interval of the second phase of tram-dummy collision. The phase started after the initial impact and finished when the tram started to brake. The maximum value reached after the initial impact and following minimum value were added

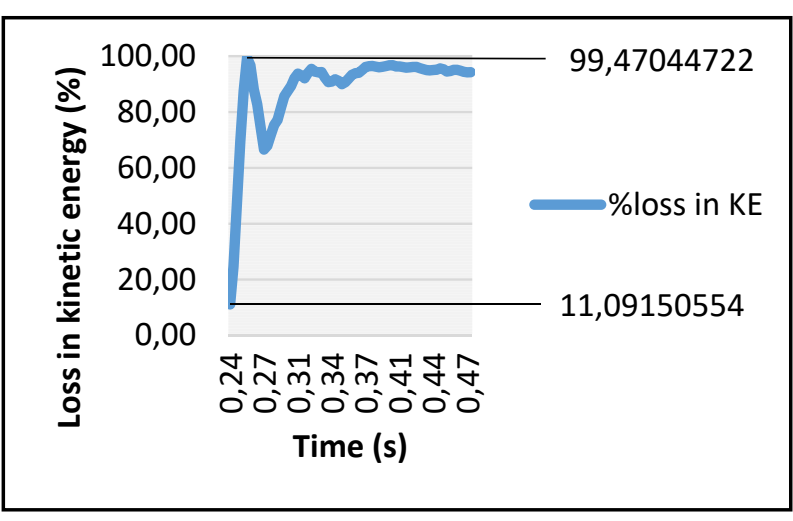

Fig. $\boldsymbol{8}$ The change of percentage loss in internal kinetic energy of the second phase of tram-dummy collision. The phase started after the initial impact and finished when the tram started to

brake. The minimum value after the initial impact and following maximum value were added

Figure 7 and 8 shows the change of coefficient of restitution (Fig. 7) and percentage loss in internal kinetic energy (Fig. 8) for the second phase of tramdummy collision that started with the initial impact and finished when the tram started to brake.

The results showed a significant change of coefficient of restitution immediately after the initial impact (Fig. 7). At first, the coefficient of restitution was high $(e=0.94)$ followed by a sharp decrease $(e=0.07)$ within a short period of time $(t=0.02 \mathrm{~s})$. As the coefficient of restitution is related to the loss of internal kinetic energy during the collision, the percentage loss in internal kinetic energy was the lowest after the initial impact $(11.09 \%)$ followed by a sharp increase reaching the highest value $(99.47 \%)$. After that, the coefficient of restitution, as well as the percentage loss in internal kinetic energy, reached a plateau phase with the values ranging from 0.18 to 0.32 , which corresponded to a percentage loss of kinetic energy falling 
within $89 \%$ and $97 \%$ during the collision.

The results, therefore, suggested that the collision between the dummy and tram was almost perfectly elastic right after the initial impact followed by a quick change into a mostly inelastic collision with a high loss of internal kinetic energy. This could be caused by the elastic properties of dummy's body parts at first, reaching their elastic limit in a short period of time, which could then result in some kind and level of deformation. However, these results need to be verified by conducting more measurements of this type of tram-dummy collision, and then also testing different impact velocities, tram types, and positions of the dummy in front of the tram. The future studies should also be focused on the analysis of the whole dummy movement after the initial impact and including the translational and rotational movements of all parts of the dummy's body.

\section{Conclusion}

The preliminary analysis of tram-dummy collision in case of a side impact highlighted some of the features based on the kinematics of colliding objects. The coefficient of restitution was found to be a useful parameter to describe the loss of internal kinetic energy during the collision that could result in deformation and possibly injury. The study outcomes could be an important step towards understanding and improving the reconstruction of traffic accidents, describing cause, type and severity of injuries, as well as assisting in the future design and development of novel technologies to improve the pedestrian safety.

\section{Acknowledgements}

The study was funded by Operational Programme Research, Development and Education CZ.02.1.01/0.0/0.0/16_026/0008401.

\section{References}

[1] PETRESCU, L., PETRESCU, A.I. (2017). Vehicle-pedestrian collisions - Aspects regarding pedestrian kinematics, dynamics and biomechanics. In: IOP Conf. Ser.: Mater. Sci. Eng., Vol. 252, 012001. DOI: 10.1088/1757$899 \mathrm{X} / 252 / 1 / 012001$.
[2] TEMG, T.L., LIANG, C.C., HSU, C.Y., and TAI, S.F. (2017). Kinematic responses and injuries of pedestrian in carpedestrian collisions. In: IOP Conf. Ser.: Mater. Sci. Eng., Vol. 248, 012029. DOI: $\quad 10.1088 / 1757-$ 899X/248/1/012029.

[3] LOPOT, F., KUBOVÝ, P., JELEN, K., ŠORFOVÁ, M., TLAPÁKOVÁ, E., RULC, V., PURŠ, H., JEŽDÍK, R., SVOBODA, M. (2019). Collision between a Pedestrian and Tram - Pilot Experiment. In: Manufacturing Technology, Vol. 19, No. 6, pp. 998 - 1002. DOI: 10.21062/ujep/409.2019/a/12132489/MT/19/6/998.

[4] BITTNER, V., JEŽDÍK, R., KUBOVÝ, P., LOPOT, F., ŠTOČEK, O., HAVLÍČEK, M., SVOBODA, M., JELEN, K. (2019). Possibilities of Using Tram Windscreen Impact Tests in Analysis of Human-Machine Accidents. In: Manufacturing Technology, Vol. 19, No. 6, pp. 912 - 916. DOI: 10.21062/ujep/395.2019/a/12132489/MT/19/6/912.

[5] Proceedings of the conference PASSIVE SAFETY of RAIL VEHICLES 2019. IFV Bahntechnik e.V. Berlin, 2019. Available at https://www.ifv-bahntechnik.de/literatur.pdf.

[6] VANGI, D. (2020). Vehicle collision dynamics: Chapter 5 - Crash analysis and reconstruction. In: Analysis and reconstruction, pp. 157 - 191. ISBN 9780128127506.

[7] JEŽDÍK, R., KONOPÍK, P., RUND, M., SVOBODA, M., JELEN, K. (2021). Determination of Material Properties of Laminates of 15T and T3 Tram Faces. In: Manufacturing Technology, Vol. 21, No. 3, pp. 349 -356. DOI: 10.21062/mft.2021.040.

[8] RICARDO, B., LEE, P. (2015). Maximizing kinetic energy transfer in one-dimensional manybody collisions. In: European Journal of Physics, Vol. 36, No. 2, pp. 1 - 12. DOI: 10.1088/0143$0807 / 36 / 2 / 025013$. 